\title{
Optical Spectra of Phytoplankton Cultures for Remote Sensing Applications: Focus on Harmful Algal Blooms
}

\author{
Robert A. Warner and Chunlei Fan
}

\begin{abstract}
Hyperspectral remote sensing reflectance was measured for a series of phytoplankton cultures as the first step in determining major taxon in an algal bloom by remote sensing Two common bloom-forming species: Dinophyta, the dinoflagellate Prorocentrum minimum, and Cyanophyta, the cyanobacteria Synechococcus sp. were grown as mono cell cultures. Optical spectral measurements were taken from the cultures during logarithmic growth phases with progressive dilutions and culture mixtures. The primary objective of this study was to obtain base line reflectance spectra which can be used as references for remote sensing of algal blooms. Furthermore, the derivative analysis was applied to the reflectance spectra to explore the spectral features that can be used to identify phytoplankton taxon. Results showed that spectral reflectance correlated with phytoplankton biomass. Applying mathematical operators to the spectra of mono cell cultures corresponded to observed spectra of culture mixtures. Our results further corroborate previous findings that for some cases, remote sensing reflectance (Rrs) can be used to identify the primary taxon in algal blooms.
\end{abstract}

Index Terms-Hyperspectral spectra, remote sensing, algal bloom, derivative analysis.

\section{INTRODUCTION}

Harmful algal blooms (HABs) occurs world widely in fresh and marine waters, which lead to serious consequences such as fisheries closures and human health concerns [1]. Large expenditures of time and money have been devoted to the monitoring and mitigation of harmful algal blooms [2]. In situ sampling sensors on platforms such as ships, autonomous underwater vehicles (AUVs), and buoys are commonly used for HABs detection in localized areas with a typical high cost [3], [4]. Remote sensing techniques are routinely used to detect blooms, typically defined by anomalously high chlorophyll values. There are ongoing efforts to refine the monitoring of HABs into determining the dominant taxa of the bloom. Simis et al. [5] used the strong absorption of phycocyanin as a bioindicator of cyanobacteria dominated blooms. Kutser et al. [6] clearly showed the placement of specific optical wavelength bands on the multi-spectral sensor MERIS (medium resolution imaging spectrometer) mounted on ESA's ENVISAT enables routine monitoring of phytoplankton blooms and identifying if the blooms are

Manuscript received November 10, 2012; revised January 10, 2013. This work was supported in part by the U.S. National Science Foundation Grant \#GEO 09871234 to C. Fan.

Robert A. Warner is with the National Oceanic and Atmospheric Administration, Center for Coastal Monitoring and Assessment, Silver Spring, MD 20910 USA (e-mail: Robert.A.Warner@noaa.gov).

Chunlei Fan is with the Biology Department/Estuarine Research Center at Morgan State University, Baltimore, MD 21251 USA (e-mail: Chunlei.fan@morgan.edu). cyanobacteria dominated. Knowing the major organism composing a bloom will enable environmental managers to make informed decisions pertaining to the bloom [7]. If the bloom organism was suspected to be of the harmful algae bloom category, shellfish bed closures may be warranted, or in some cases beach closures to lessen inhalation of aerosols by humans. Remote sensing techniques provide the synoptic monitoring capability and could provide information of the phytoplankton taxon responsible for the algal blooms.

The objective of this study is to measure and characterize the reflectance spectra for several bloom forming phytoplankton species in the Chesapeake Bay, US. We further explore the spectral signatures from these cultures which could be further applied as reference spectra to field and airborne optical measurements to indentify the major phytoplankton groups in the algal blooms.

\section{MethodS AND MATERIALS}

\section{A. Phytoplankton Culture and Spectral Measurement}

The dinoflagellate, Prorocentrum minimum, was grown in $\mathrm{F} / 2$ plus soil extracting media, whereas the cyanobacteria, synechococcus sp., was grown in F/2 media without additional media. Mono cell cultures were grown in $20 \mathrm{~L}$ clear polyethylene carboy at $20^{\circ} \mathrm{C}$, under a 12 hour light and 12 hour dark cycle. Cultures at exponential growth phase were transferred to plastic flat black cans $(50 \mathrm{~L})$, filled to the brim for spectral measurements. The weather conditions during the measurement were clear, with low humidity. A Field Spec portable spectroradiometer (ASD Inc.) was employed for the optical measurements and a one degree fore-optic was used to minimize optical contamination. Sun-to-sample plane was kept about 90 degrees (nadir), and all measurements were made within $3.5 \mathrm{~h}$ of solar noon. The ASD spectroradiometer first measured the down welling incident irradiance $\left(\mathrm{E}_{\mathrm{d}}\right)$ from a $10 \%$ calibrated reflectance Spectralon reference panel (Labsphere, Inc.), then pointed to the cultures and acquired the upwelling radiance of the samples $\left(\mathrm{L}_{\mathrm{w}}\right)$. The remote sensing reflectance (Rrs) is calculated as $\operatorname{Rrs}=\mathrm{L}_{\mathrm{w}} / \mathrm{E}_{\mathrm{d}}$ with the unit of $\mathrm{sr}^{-1}$.

Standard $\mathrm{Hg}$ and $\mathrm{Ar}$ lamps were used to verify wavelength calibration. Radiometric calibration was verified using LabSphere 8 port calibration sphere with xenon and tungsten lamps. Spectral resolution defined as full width at half maximum (FWHM) was $3 \mathrm{~nm}$ at $700 \mathrm{~nm}$. Each spectrum consisted of 5 dark current measurements, 5 reference panel measurements and 10 water leaving radiance measurements. Five spectral measurements were averaged for the final Rrs values, thus an average of 50 spectra were averaged for each reflectance measurement. The remote sensing reflectance (Rrs) from $350 \mathrm{~nm}$ to $900 \mathrm{~nm}$ was presented in this study. 
Samples for Chlorophyll a concentration, and HPLC pigments were collected by filtering onto Whatman $\mathrm{GF} / \mathrm{F}$ filters with less than $100 \mathrm{mmHg}$ vacuum for further analysis. Chlorophyll a was extracted using 90\% acetone and measured with a Spectronic Genesys II spectrophotometer (Spectronics, Inc.) [8]. HPLC pigment analysis was conducted at the University of Maryland, Horn Point Laboratory.

To further explore how the phytoplankton biomass could affect reflectance spectra, mono cell cultures were diluted 50\% $\mathrm{v} / \mathrm{v}$ with filtered seawater, and the second set of culture spectra were collected. The reflectance spectra were also measured for the mixtures $(50 \% \mathrm{v} / \mathrm{v})$ of the two phytoplankton cultures to access the impacts of different phytoplankton pigments on reflectance spectra.

\section{B. Derivative Analysis of Reflectance Spectra}

In this study, derivative analysis was applied to the measured reflectance spectra to determine the spectral features that can be used for algal taxon identification. Derivative analysis has been successfully used to detect individual pigment signatures in overlapping absorbance spectra [9], and more recently, in radiance spectra [10]. It has been suggested that derivative analysis can identify contributions from different classes of micro algae, such as using accessory pigments of chlorophyll ${ }^{\mathrm{b}}$ for green algae, fucoxanthin for diatoms, and peridinin for dinoflagellates [11], [12]. For most cases, the fourth derivative had more pronounced spectral features for these algal pigments and thus resolved components more than the second derivative [13].

The fourth derivative analysis was conducted for all measured reflectance spectra (Rrs) by a software package Viewspec Pro (ASD Inc.). The spectral gap was $20 \mathrm{~nm}$ in the derivative calculation.

\section{RESULTS}

\section{A. HPLC Pigment Analysis}

High-performance liquid chromatography (HPLC) provided clear distinction for the two classes of phytoplankton in this study. Marra et al. [14] divides phytoplankton pigments into three groups: 1. pigments directly responsible for photochemical energy conversion (chlorophyll ${ }^{\mathrm{a}}$ ), absorbing at $410 \mathrm{~nm}, 430 \mathrm{~nm}$ and $662 \mathrm{~nm} ; 2$. pigments that transfer photons to chlorophyll ${ }^{\mathrm{a}}$ (chlorophyll ${ }^{\mathrm{b}}$, absorbing at $453 \mathrm{~nm}$ and $642 \mathrm{~nm}$, chlorophyll ${ }^{\mathrm{c}}$, carotenoids, phycobilins, absorbing $550 \mathrm{~nm}$ to $650 \mathrm{~nm}$ ); and 3 . photoprotectants, another function of carotenoids, absorbing in the 400-500 $\mathrm{nm}$ range. This information of pigment composition can be useful in interpreting spectra.

The predominant pigments measured for Synechococcus sp. by HPLC were carotene $(22.0 \mathrm{ug} / \mathrm{L})$, zeaxanthin $(99.3$ $\mathrm{ug} / \mathrm{L})$, and phaeophytina (70.0 ug/L), where chlorophylla was $29.9 \mathrm{ug} / \mathrm{L}$, while the HPLC analysis for the dinoflagellate Prorocentrum minimum had high total chlorophyllc (289 $\mathrm{ug} / \mathrm{L})$, diadinoxanthin (178 ug/L), peridininol (470 ug/L), viola $(1.7 \mathrm{ug} / \mathrm{L})$, phidea (44 ug/L) and chlorophyllc2 (289 ug/L), where total chlorophylla was $785 \mathrm{ug} / \mathrm{L}$. So, beside of total chlorophylla, zeaxanthin and phaeophytina were the dominated pigment for Synechococcus sp., and peridininol were the dominated pigment for Prorocentrum minimum.

\section{B. Reflectance (Rrs) Spectra for Mono Cell Culture}

The reflectance (Rrs) spectra and the fourth derivative of these spectra of mono cell cultures are showed in Fig. 1 Synechococcus sp. and in Fig. 2 for Prorocentrum minimum. The "red edge" $(680 \mathrm{~nm}$ to $730 \mathrm{~nm})$ is prevalent in the spectra of both cultures. Chlorophylls reemit a fraction of the light energy they absorb as fluorescence, which is emitted in the red or infrared region of the spectrum resulting in the observed slope in the near infra-red region [15].

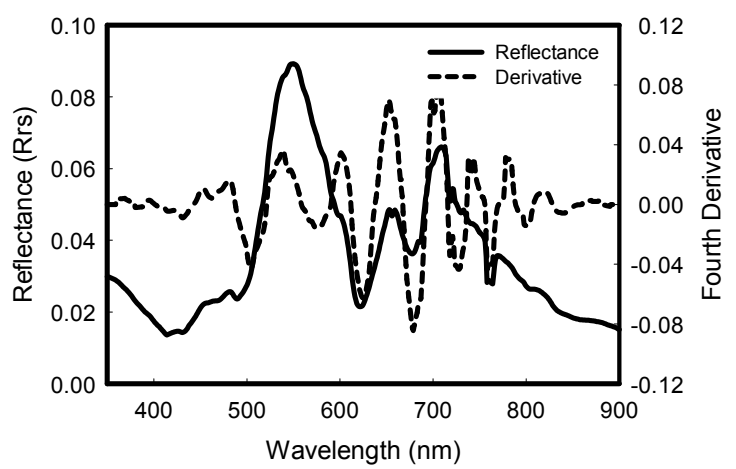

Fig. 1. The reflectance (Rrs) spectra and the fourth derivation of the reflectance (Rrs) for Synechococcus sp.culture.

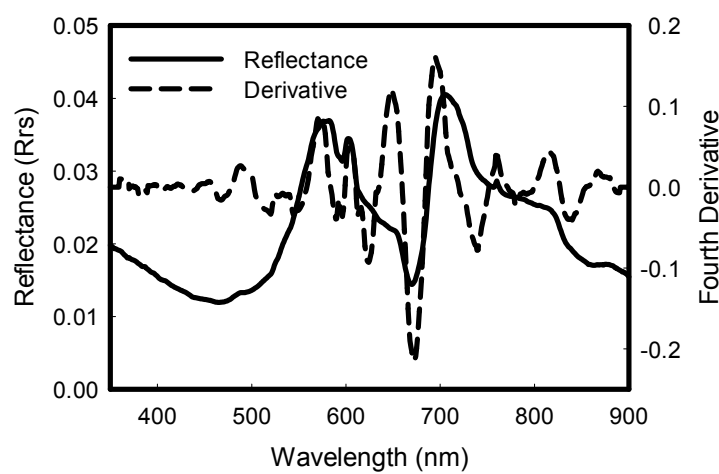

Fig. 2. The reflectance (Rrs) spectra and the fourth derivative of the reflectance (Rrs) for Prorocentrum minimum culture.

The Synechococcus sp. (Fig. 1) cultures showed the well described absorption feature at $622 \mathrm{~nm}$ due to absorption of phaeophytina and a minor reflection peak at $651 \mathrm{~nm}$. The fourth derivative showed the reflectance peak at $653 \mathrm{~nm}$ with a shoulder at $656 \mathrm{~nm}$, however the source of the feature is unknown. There were many other features seen in the Rrs spectra and enhanced by the $4^{\text {th }}$ derivative analysis, however many of these pigment complexes were not part of the HPLC suite, nor found in the literature. It is interesting to note the strong absorption feature at $758 \mathrm{~nm}$, beyond the photosystem I complex region. The spectral feature at $758 \mathrm{~nm}$ may be attributed to atmospheric oxygen [15].

Other than the photosystem I region, the dinoflagellate, Prorocetrum minimum had strong spectral features (Fig. 2) at different locations of the spectra compared with the Synechococcus sp. The overall Rrs for Synechococcus ( $\mathrm{Rrs}=$ 0.092 ), especially around $550 \mathrm{~nm}$ green spectral region, were about 2 times higher than Prorocentrum $(\operatorname{Rrs}=0.037)$. This may be accounted for the difference in pigment concentrations, as well as in part by the difference in cell size dinoflagellates are much larger $(\sim 20 \mu \mathrm{m})$ and have more internal cellular structures than the prokaryotic Synechococcus $(\sim 1 \mu \mathrm{m})$. There is a strong absorption feature 
for Prorocentrum at $595 \mathrm{~nm}$ while there is no obvious absorption for Synechococcus at this wavelength. Similar, the well described absorption feature for Synechococcus at $622 \mathrm{~nm}$ due to phaeophytina was not observed in Prorocentrum spectra. Prorocentrum spectra also have a broad absorption region around $470 \mathrm{~nm}$ is likely due, in part, to the caroteoid peridinin. Finally, the absorption at $680 \mathrm{~nm}$ by Prorocentrum about two times stronger than Synechococcus, and this is likely accounted for the higher chlorophylla concentration in Prorocentrum cultures.

\section{Reflectance (Rrs) Spectra for Dilution and Mixture Experiments}

Diluting the cultures by $50 \%$ with filtered seawater resulted in an approximately $50 \%$ decrease of the maximum reflectance values (Fig. 3 and Fig. 4). Synechococcus culture dilution resulted in a decrease in Rrs from 0.086 to 0.044 at $541 \mathrm{~nm}$. The $50 \%$ dilution of Prorocentrum culture resulted in a decrease at the maximum reflectance from 0.039 to 0.022 at $700 \mathrm{~nm}$. However, this decrease of Rrs was not uniform across the measured spectral range. Only slight decreases of Rrs at shorter wavelength (350 to $450 \mathrm{~nm}$ ) were observed with dilution. We did not observe this trend for absorption features nor for spectral averages, where only slight decreases were observed with dilution. This decrease of Rrs became more pronounced at the $500-800 \mathrm{~nm}$ range. This wavelength dependent dilution effect was possibly due to fewer pigments available for absorption and the spectra of water dominating at the $350-450 \mathrm{~nm}$ region.

In the natural aquatic environment, more than one algal species could usually dominate a phytoplankton blooms. So, in this study, the two mono cell cultures were further mixed to evaluate identification of phytoplankton species from the spectra with different algal species. Overall, major spectral features for both Synechococcus and Prorocentrum were preserved in the mixture (Fig. 6). The mathematical mixture of spectra from the two pure cultures (Fig. 3 and Fig. 4) resulted in similar spectra denoting the spectral features of the culture mixture but with higher RRS values than the laboratory mixture. The difference between the math mixed and the laboratory mixture of cells may have been attributed to a dilution affect with adding the laboratory cultures as well as a possible uneven distribution of cells.

\section{Spectral Comparison to the Field Data}

Four archived spectra were obtained (Fig. 6) during three cyanobacteria blooms in the Potomac River, Maryland, US (August 18, 2008, August 28, 2006, September 18, 2006), and one in Bear Lake, Michigan, US (August 6, 2006). The blooms were at different life stages when the spectra were measured. September 18 in the Potomac and August 1 in Bear Lake were possibly at late bloom states, as noted by ecologists in the boat. Potomac River August 18 was near the peak of the bloom, where August 28 measured at later bloom stage. First of all, the absorption feature around $620 \mathrm{~nm}$ (diagnostic absorption by phycocyanins for cyanobacteria) was observed for all field spectral data, even the absorption intensity was different in these field spectral data. When compared with the spectra of Synechococcus culture, the strongest absorption was observed during the August 18 bloom in Potomac River, Maryland, when the field bloom was the peak stage. Taking the difference between the peak and trough of about $550 \mathrm{~nm}$ and about $620 \mathrm{~nm}$, we see that indeed the magnitude in decreasing order was the culture (0.066), Aug 18 (0.040), Aug 28 (0.011), Bear Lake (0.003) and Potomac September 18 (0.002). The negative slope at $681 \mathrm{~nm}$ when compared to the baseline established by 665 $\mathrm{nm}$ and $709 \mathrm{~nm}$ points (Fig. 6) corroborate the results of Wynne et al. [16] with the field and MERIS data [17].

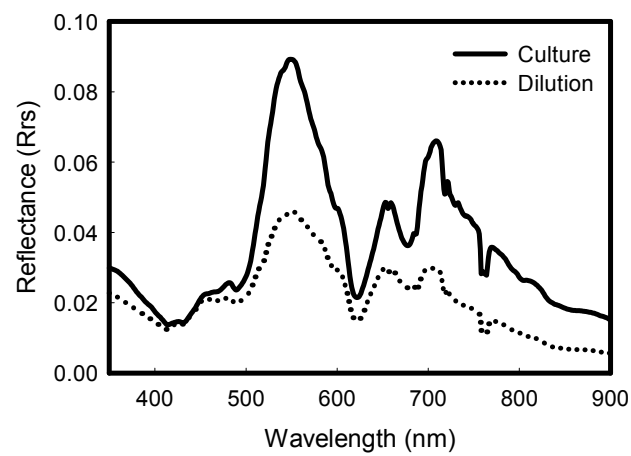

Fig. 3. The reflectance (Rrs) spectra for synechococcus sp culture and its $50 \%$ dilution culture.

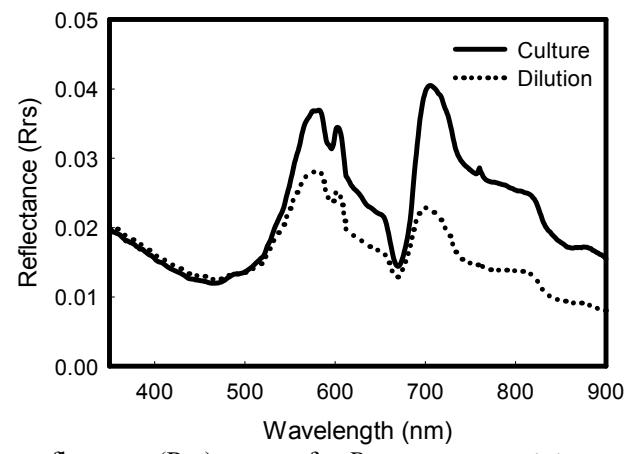

Fig. 4. The reflectance (Rrs) spectra for Prorocentrum minimum culture and its $50 \%$ dilution culture.

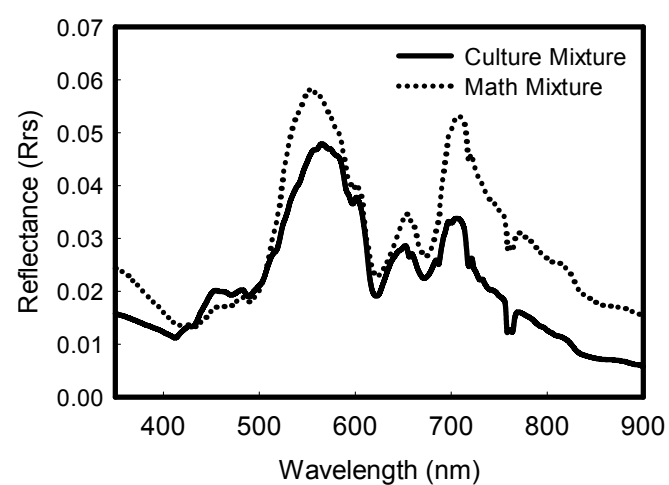

Fig. 5. The reflectance (Rrs) spectra for the mixing (50:50) cultures of Synechococcus sp. and Prorocentrum minimum. And the mathematical mixture of spectra from the two pure cultures.

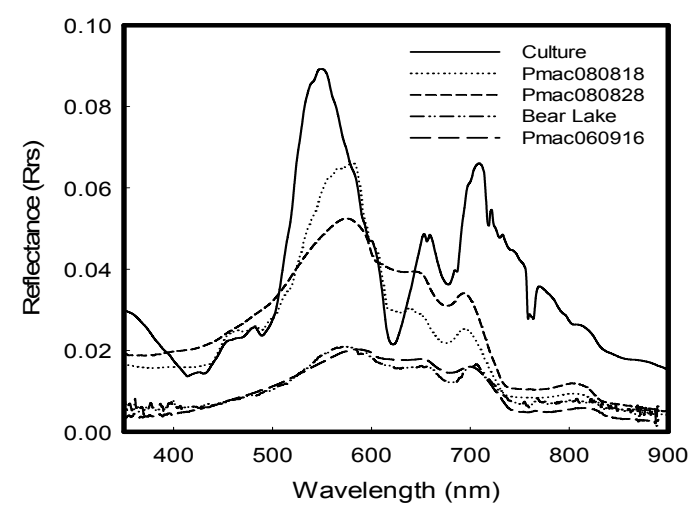

Fig. 6. The reflectance (Rrs) spectra for achieved cynaobacteria field data and the culture of Synechococcus sp. 


\section{Discussion}

Remote sensing has been proposed as a means of synoptically monitoring, detecting, and characterizing the location and extent of harmful algal blooms, and is becoming increasingly relied on by researchers and coastal management agencies [18]. Using the reflectance spectra from mono cell cultures can provide the direct comparison with what the airborne and space borne sensor are detecting (e.g. the remote sensing reflectance, Rrs), and thus can assist in developing algorithms to process remotely sensed data for HABs detection. However, these reflectance data are not extensively available for such purpose. In general, the whole cell absorption spectra (not the reflectance data, e.g. Rrs), where the spectra features are associated with contributing molecules or pigments [19] are used toward to the understanding and interpretation of remote sensing spectra. This study is the initial step to build a reflectance spectral library with radiance and reflectance (Rrs) typically used in remote sensing, which can lead to the algorithms in detecting major phytoplankton groups in a harmful algal blooms.

Our approach is to use natural light to obtain reflectance data from pure cultures, as well as from laboratory manipulated cell mixtures and dilutions. These spectra will be used to construct a spectral library of reference organisms, or standards, and to later apply these reference data to airborne imagery to test our laboratory determined analytical methods to identify specific micro algal groups. This is in contrast to previous work, which took imagery and successfully separated out unique end members, which were then identified from field collections [20]. Other studies directly measured absorption at discrete wavelengths of in situ samples and applied analysis techniques to those data [21].

However, the approach of direct measurement of remote sensing reflectance (Rrs) that was used in this study could be influenced by some environmental factors, such as the sky condition and the light contaminations from the containers and the surrounding environments [22]. In this study, the measurements were carefully carried out in a clear day with low humidity. The containers for mono cell cultures were black and non reflective to minimize the wall effect. A one degree fore-optic was used to minimize optical contamination from the surrounding environments. Additional measures were taken to test for optical contamination by observing and analysis of water spectra taken from de-ionized water in a clean sample container. There were no observed optical contaminations (data not shown here). Reflectance spectra of the water were comparable with published data [23], [24]. Fourth derivative analysis of the water spectra show not significant spectral features, which further confirmed the absence of optical contamination for these measured reflectance spectra (Rrs).

Combined with the HPLC pigment analysis, the data presented in this study is the initial step to demonstrate the potential usefulness of hyperspectral remote sensing reflectance for discriminating different phytoplankton assemblages in laboratory and field conditions. The two mono cell cultures have spectral features that can be distinguished in individual spectra as well as in the culture mixture, and these spectral features were closely related to the pigment composition of the two classes of phytoplankton in the PHLC analysis. The Cyanophyta (blue green algae or cyanobacter) contained bioindicators of zeaxanthin and phaeophytin $^{\text {a }}$, Where the Dinophyta (din flagellates) had bioindicators of total chlorophyll ${ }^{\mathrm{c}}$, peridinin, and phaeophorbide $^{a}$ [25]. Furthermore, these spectral features were preserved no matter in the dilution and mixing manipulations. Our preliminary data has been comparable with few similar studies [26], which suggested the detection and mapping of some algal blooms based on hyperspectral measurements of remote-sensing reflectance appear to be feasible.

The derivative analysis of the remote sensing reflectance provided a better discrimination for the spectral features, such as the fourth derivative show a prominent absorption feature at $622 \mathrm{~nm}$ due to absorption of phaeophytin ${ }^{\mathrm{a}}$ for culture of Synechococcus sp., while the absorption feature due to peridinin for Prorocentrum minimum culture can be observed at $595 \mathrm{~nm}$. Fourth derivates with a derivative gap of $21 \mathrm{~nm}$ were applied to the field achieve data measured during several cyanobacteria blooms (Fig. 7). The analysis enhanced the major features of the spectral reflectance (e.g absorption around $620 \mathrm{~nm}$ and $680 \mathrm{~nm}$ ). Some subtle absorption features around $500 \mathrm{~nm}$ were also detected, however, the pigments associated with these subtle absorption features can't be identified. This may be due to the large range of pigment compositions across different phytoplankton classes. Determinations of phytoplankton composition from HPLC pigment data is not straightforward [27]. While certain diagnostic pigments can serve as unambiguous markers for some phytoplankton classes, many important pigments are shared by more than one algal taxas (e.g., fucoxanthin in diatoms, haptophytes, chrysophytes, and raphidophytes). Nevertheless, because many of the classes have distinctive suites of marker pigments, HPLC data with the derivative analysis can be useful for indicating their presence and abundance in a mixed phytoplankton population. The findings in this study also corroborate the general characteristics of the reflectance spectra found by Gitelson et al. [28] and Craig et al. [24] that used derivative analysis to assess the feasibility of detection of a toxic bloom of the dinoflagellate Karenia breve from the analysis of the fourth derivative of phytoplankton reflectance spectra. We intend on employing a similarity index to our fourth derivative spectal analyses as was successfully utilized for Gymnodinium breve [27].

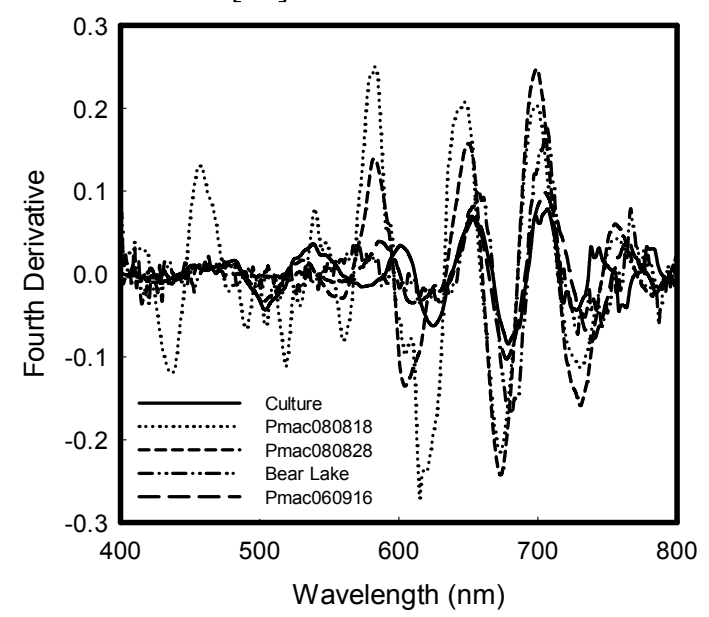

Fig. 7. The fourth derivative of the reflectance (Rrs) spectra for achieved cynaobacteria field data and the culture of Synechococcus $s p$. 
It is known that phytoplankton undergo metabolic changes when stressed, such as different levels of light, nutrients, salinity, temperature, and carbon dioxide. Light harvesting pigment complexes can change dramatically [28]. We intend to continue this study to optically assess spectral features with changes of pigment when algal cells undergo a matrix of stress conditions, as well as the influences of other water optical constituents, such as suspended sediment, and colored dissolved organic matters (CDOM). These future works are expected to provide more information for the feasibility of using remote sensing to detect and map the harmful algal blooms at the regional scale.

\section{ACKNOWLEDGMENT}

We would like to thank the assistance from Morgan State University Estuarine Research Center, AnnMarie Hartsig for assistance with growing the phytoplankton cultures and cell counts, Richard V. Lacouture for allowing above surface spectra to be taken during his water quality boat survey program in the Chesapeake Bay region, and to the University of Maryland, Horn Point laboratory for HPLC analysis.

\section{REFERENCES}

[1] T. J. Smayda, "What is a bloom? A commentary," Limno. Oceanogr vol. 42, no. 5, pp. 1132-1136, June 1997.

[2] D. M. Anderson, P. M. Glibert, and J. M. Burkholder, "Harmful algal blooms and eutrophication: Nutrient sources, composition, and consequences," Estuaries, vol. 25, no. 4b, pp. 562-584, May 2002.

[3] T. S. Platt, S. Sathyendranath, C. M. Caverhill, and M. R. Lewis, "Ocean primary production and available light: further algorithms for remote sensing," Deep Sea Res., vol. 35, pp. 855-879, 1988.

[4] F. E. Muller-Karger, J. Walsh, R. H. Evans, and M. B. Meyers, "On the seasonal phytoplankton concentration and sea surface temperature cycles of the gulf of Mexico as determined by satellites," J. Geophys. Res., vol, 96, pp. 12645-12665, 1991.

[5] S. G. H. Simis, S. W. M. Peters, and H. J. Gons, "Remote sensing of the cyanobacterial pigment phycocyanin in turbid inland water," Limno. Oceanogr., vol. 50, no. 1, pp. 237-245, 2005.

[6] T. Kutser, L. Metsamaa, N. Strombeck, and E. Vahtmae, "Monitoring cyanobacterial blooms by satellite remote sensing," Coastal and Shelf Sci., vol. 67, pp. 303-312, 2005.

[7] P. D. Hunter, A. N. Tyler, M. Presing, A. W. Kovacs, and T. Preston, "Spectral discrimination of phytoplankton colour groups: The effect of suspended particulate matter and sensor spectral resolution," Remote Sens. of Environm, vol.112, pp. 1527-1544, 2008.

[8] R. Mitchell (ed.), Standard Methods for the Examination of Water and Wastewater, $17^{\text {th }}$ ed., pp. 1527, American Public Health Association, Washington D.C., 1989.

[9] R. R. Bidigare, J. H. Morrow, and D. A. Kiefer, "Derivative analysis of spectral absorption by photosynthetic pigments in the western Sargasso Sea," J. Mar. Res., vol. 47, pp. 323-341. 1989.

[10] L. L. Richardson and F. A. Kruse, "Identification and classification of mixed phytoplankton assemblages using AVIRIS image-derived spectra," presented at AVIRIS workshop, JPL Pasadena, CA, June 2-6, 2000.

[11] K. Suzuki, M. Kishino, K. Sasaoka, S. Saitoh, and T. Saino, "Chlorophyll-specific absorption coefficients and pigments of phytoplankton off Sanriku, northwestern north Pacific," J. Oceanogr., vol. 54, pp. 517-526, 1998.

[12] G. J. Kirkpatrick, D. F. Millie, M. A. Moline, and O. Schofield, "Optical discrimination of a phytoplankton species in natural mixed populations," Limno. Oceanogr, vol. 45, no. 2, pp. 467-471, 2000.
[13] W. L. Butler and D. W. Hopkins, "Higher derivative analysis of complex absorption spectra," Photochem. Photobio., vol. 4, pp. 439-450, 1970.

[14] J. Marra, C. C. Trees, R. R. Bidigare, and R. T. Barber, "Pigment absorption and quantum yields in the Arabian Sea," Deep Sea Res., vol. 47, no. 2, pp.1279-1299, 2000

[15] G. D. Greenblat, J. J. Orlando, J. B. Burkholder, and A. R. Ravishankara, "Absorption measurement of oxygen between 330 and 1140 nm," J. Geophy. Res., vol. 95, no. 11, pp. 18,577 - 18,582, 1990

[16] T. T. Wynne, R. P. Stumpf, M. C. Tomlinson, R. A. Warner, P. A. Tester, J. Dyble, and G. L. Fahnenstiel, "Relating spectral shape to cyanobacterial blooms in the Laurentian Great Lakes," Int. J. Rem. Sens., vol. 29, no. 12, pp. 3665-3672, 2008.

[17] T. T. Wynne, R. P. Stumpf, M. C. Tomlinson, and J. Dyble, "Characterizing a cyanobacterial bloom in Western Lake Erie using satellite imagery and meteorological data," Limno. Oceanogr., vol. 55, no. 6, pp. 1397-1407, 2010.

[18] Govindjee and B. Z. Braun, "Light absorption, emission and photosynthesis," Algal physiology and Biochemistry, W. D. P. Stewart, Ed. University of California press, pp. 989, 1974.

[19] D. E. Millie, O. M. Schofield, G. J. Kirkpatrick, G. Johnsen, P. A. Tester, and B. T. Vinyard, "Detection of harmful algal blooms using photopigments and absorption signatures: A case study of the Florida red tide dinoflagellate, Gymnodinium breve," Limno. Oceanogr., vol. 42, no. 5, pp. 1240-1251, 1997

[20] R. M. Pope and E. S. Fry, "Absorption spectrum (380-700 nm) of pure water. II. Integrating cavity measurements," Appl. Optic., vol. 36, pp. 8710-8723, 1997.

[21] S. Sathyendranath, V. Stuart, G. Cota, H. Maass, and T. Platt, "Remote sensing of phytoplankton pigments: a comparison of empirical and theoretical approaches," Int. J. Rem. Sens., vol. 22, pp. 249-273, 2001

[22] R. P. Stumpf, M. E. Culver, P. A. Tester, M. Tomlinson, G. J. Kirkpatrick, B. A. Pederson, E. Truby, V. Ransibrahmanakul, and M. Soracco, "Monitoring Karenia brevis blooms in the Gulf of Mexico using satellite ocean color imagery and other data," Harmful Algae, vol. 2, pp. 147-160, 2003.

[23] T. G. Owens, J. C. Gallagher, and R. S. Alberte, "Photosynthetic light-harvesting function of violaxanthin in nannochloropsis spp. (Eustigmatophyceae)," J. Phyco., vol. 23, no.1, pp. 79-85, 2007.

[24] S. E. Craig, S. E. Lohrenz, Z. P. Lee, K. L. Mahoney, G. J. Kirkpatrick, O. M. Schofield, and R. G. Steward, "Use of hyperspectral remote sensing reflectance for detection and assessment of the harmful alga, Karenia brevis," Appl. Optics. vol. 45, no. 21, 2006.

[25] S. W. Jeffrey, S. W. Wright, and M. Zapata, "Recent advances in HPLC pigment analysis of phytoplankton," Mar. Freshwater Res., vol. 50, pp. 879-896, 1997.

[26] A. A. Gitelson, J. F. Schalles, D. C. Rundquist, F. R. Schiebe, and Y. Z. Yacobi, "Comparative reflectance properties of algal cultures with manipulated densities," J. Appl. Phycol., vol. 11, pp. 345-354, 1999.

[27] G. J. Kirkpatrick, D. F. Millie, M. A. Moline, and O. Schofield, "Optical discrimination of a phytoplankton species in natural mixed populations," Limnol. Oceanogr., vol. 45, no. 2, pp. 467- 471, 2000.

[28] J. Marra, C. C. Trees, R. R. Bidigare, and R. T. Barber, "Pigment absorption and quantum yields in the Arabian Sea," Deep Sea Res., vol. 47, no. 2, pp.1279-1299, 2000.

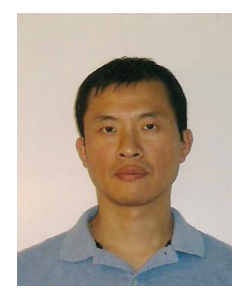

Dr. Chunlei Fan is currently working as an associate professor at Estuarine Research Center/Biology Department, Morgan State University in Baltimore, Maryland, USA. He has completed his B.S in environmental sciences at Shandong University in 1991, and M.S in oceanography at Institute of Oceanography, Chinese Academy of Sciences, in 1995. He completed his Ph.D in environmental science at University of Mar yland Collage Park in 2002. Studying the human impacts on the coastal water and monitoring eutrophication process have been his primary research interests. He is a member of American CERF and ASLO. 